\title{
Risk of total knee replacement after proximal tibia fracture: a register-based study of 7,701 patients
}

\author{
Valtteri S TAPPER ${ }^{1}$, Konsta J PAMILO ${ }^{2}$, Jaason J HAAPAKOSKI ${ }^{3}$, Alar TOOM ${ }^{1}$, \\ and Juha PALONEVA 1,4
}

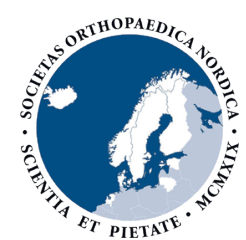

Acta Orthopaedica

\author{
${ }^{1}$ Central Finland Health Care District, Department of Surgery, Jyväskylä; ${ }^{2}$ Coxa Hospital for Joint Replacements, Tampere; \\ ${ }^{3}$ National Institute for Health and Welfare (THL); ${ }^{4}$ University of Eastern Finland, Kuopio, Finland \\ Correspondence: valtteri.tapper@fimnet.fi \\ Submitted 2021-02-25. Accepted 2021-11-07.
}

Background and purpose - Post-traumatic knee osteoarthritis following proximal tibia fracture (PTF) is a common complication that may lead to total knee replacement as secondary treatment (TKRS). We determined the risk of TKRS following PTF, whether treated nonoperatively or operatively, and compared the results with a 38 -fold control group without prior PTF.

Patients and methods - We identified all patients over 18 years of age in Finland with PTF treated during the period 2009-2018 from the Finnish Hospital Discharge Register (FHDR) and Finnish Arthroplasty Register (FAR). Age, sex, treatment method, follow-up time, and possible TKRS were recorded.

Results $-7,701$ patients were treated for PTF during the period 2009-2018. Over the 5.1-year (SD 3.1) follow-up, TKRS was performed in $340(4.3 \%)$ patients with a prior PTF after a mean of 2.1 (SD 2.0) years post-fracture. TKRS was needed in 138 (3.7\%, HR 1.8) patients in the nonoperatively treated group and in $202(5.0 \%$, HR 3.2) patients in the operatively treated group. Operative treatment, female sex, and high age were identified as risk factors for TKRS. The incidence of TKRS was highest during the first 2 years after fracture and remained elevated throughout the follow-up.

Interpretation - Patients with a prior PTF had a 1.8- to 3.2-fold higher risk of TKRS compared with controls during the first 5 years post-fracture. Risk of TKRS was associated with an operatively treated PTF, female sex, and high age. The patients in the operative group likely sustained more complex fractures, while female sex and age may be explained by more osteoporotic bone quality.
Knee fractures cause long-term disability and increased mortality (1). The incidence of proximal tibia and tibial plateau fracture varies between 10 and 27/100,000 and rises with age. Older women in particular are at higher risk (2-4).

Proximal tibia fractures (PTF) are treated operatively with open reduction and internal fixation (ORIF) or nonoperatively with plaster or orthosis. Nonoperative treatment is used in cases of minimally displaced fractures, non-ambulatory patients, and patients with severe comorbidities. For displaced PTFs leading to joint instability, operative treatment is preferred. Following both treatment modalities, post-traumatic knee osteoarthritis (OA) is common. Among operatively treated patients with intra-articular knee fractures, 21-75\% develop post-traumatic OA even when adequate reduction and stable fixation have been achieved $(\mathbf{5 , 6 )}$. Risk factors reported for secondary OA are high age and osteoporotic bone quality, comorbidity, malalignment of the knee, articular collapse, and fracture comminution $(\mathbf{5 , 7 , 8 )}$. The rate of OA development after a nonoperatively treated PTF is unknown, but may be somewhat lower than after operatively treated fractures (9).

However, the reported rates of total knee replacement as secondary treatment (TKRS) after PTF are relatively low. Severe OA leading to TKRS typically develops in $2.2-7.5 \%$ of operatively treated patients after a median of 2 to 5 years post-trauma (8-11). In comparison with a normal population, these patients are at a 3.5- to 5.3-fold higher risk of end-stage post-traumatic OA requiring TKRS $(\mathbf{8 , 9})$.

The primary aim of this study, which included the whole population of Finland, was to determine the risk of TKRS following prior PTF treated both nonoperatively and operatively and compare the results with the corresponding risk in a 38-fold control group without PTF. Secondary aims of the study were to ascertain if the risk of TKRS is associated with patient sex or age. 


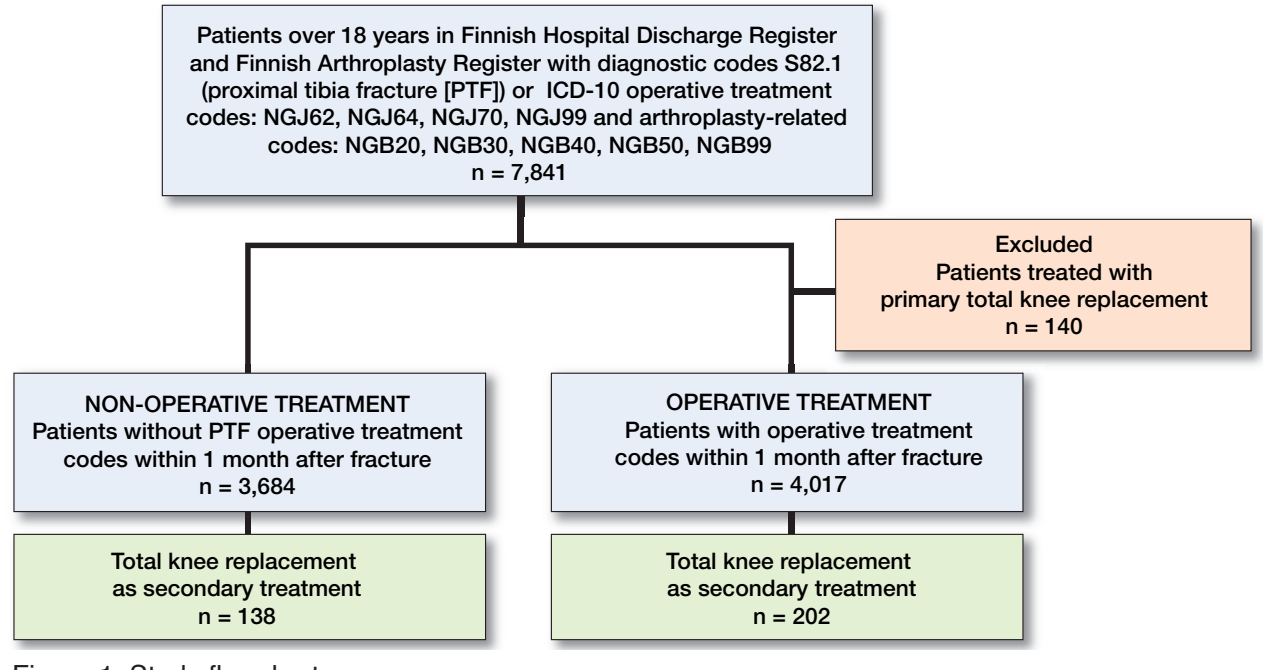

Figure 1. Study flowchart.

\section{Patients and methods}

National registry data was retrospectively collected from the Finnish Hospital Discharge Register (FHDR) and Finnish Arthroplasty Register (FAR). All public and private hospitals in Finland report all surgical procedures to the FHDR and arthroplasties to the FAR. The validity of the FHDR has been found to be good in both data coverage and accuracy (12). The coverage of FAR during the period 2009-2018 was 94\% of all the knee arthroplasties performed in Finland (https://www. thl.fi/far/\#data/cphd). The data collected from both registries included age, sex, time of fracture, time to possible TKRS, and date of death. The follow-up time was counted from the date of diagnosis to the date of death, the date of TKRS, or to the end of the study follow-up in March 2020.

All patients over 18 years, who sustained a PTF in Finland during the period 2009-2018, were included in the study. The study population was extracted by a computer-assisted search of the FHDR using the diagnosis code for PTF (ICD-10: S82.1) and the surgical procedure codes for tibia fractures and total knee replacement (NOMESCO code, Finnish version, ICD-10: NGJ62, NGJ64, NGJ70, NGJ99, NGB20, NGB30, NGB40, NGB50, NGB99; see Supplementary data). The patients with PTF drawn from the FHDR were then screened using the FAR to identify those who later underwent TKRS. To capture all patients and to assess the coverage of FAR, the data in FAR and FHDR on PTF was combined and duplicates removed. Information on the laterality of the PTFs and TKRS was not recorded in the FHDR, thus introducing a risk of observations on sides without a prior fracture. To estimate this risk, a control group of 620 patients with known laterality of the PTF and TKRS was formed from our hospital database for the period 1998-2017.

Patients with a surgical procedure code for a PTF operation within 1 month after fracture formed the operatively treated group. Patients who had no fracture-related operations or an operation at least 1 month after fracture formed the nonop- eratively treated group. If the TKR operation was performed within 2 weeks after fracture, it was categorized as primary TKR, and these patients $(n=140)$ were excluded. TKR operations performed more than 2 weeks after fracture were categorized as TKRS. The 2-week breakpoint labeling for primary TKR was based on own hospital data (all primary TKR operations were performed within 2 weeks after fracture) (Figure 1). Patients with intraoperative fractures during the TKR procedure were excluded.

To compare TKR risk in patients with PTF with that of a population without prior PTF, a reference group comprising patients with diagnosis code R10.4 for unspecified stomach pain, one of the most common diagnoses in the register, was drawn from the FHDR. The diagnosis is common in all ages and is unlikely to be related to PTF and TKRS. The control group comprised 298,397 patients with a mean follow-up of 5.1 years.

To calculate fracture incidence, the annual population $(>18$ years old) of Finland was derived from the Statistics Finland website (https://www.stat.fi/index_en.html) and distributed by age and sex. The crude age- and sex-specific incidence rates were calculated per 100,000 person-years.

\section{Statistics}

We used the Kaplan-Meier method to estimate the proportions of patients requiring TKRS after PTF in the case (operative treatment/nonoperative treatment, men/women) and reference groups. We used a non-stratified Cox regression model to estimate hazard ratios for TKRS after PTF compared with the reference group without PTF, using the following covariates: sex, age (continuous), and the initial method of treating TPF (operative/nonoperative). Due to possible issues with the proportional hazards assumptions, we also fitted a Cox model stratified by treatment group and estimated cumulative treatment effects for initial treatment (operative vs. nonoperative) in the PTF cohort and for nonoperatively treated PTF compared with the control group, according to the method described by Wei and Schaubel (13). Model fit was evaluated by the concordance index and Schoenfeld residuals. Ratios with 95\% confidence intervals (CI) were plotted for all follow-up events (TKRS, end of follow-up time without TKRS, or death) at each follow-up time point.

The R "survival" package (R Foundation for Statistical Computing, Vienna, Austria) was used in the analysis: the "survfit" function for the crude Kaplan-Meier estimation, and the "coxph" function for the Cox model. Schoenfeld residuals were calculated by the function "ggcoxzph" in the R "survminer" package. 
Table 1. Patients in the study. Values are count (\%) unless otherwise specified

\begin{tabular}{lccc}
\hline & & \multicolumn{2}{c}{ Treatment } \\
Factor & Total & Operative & Non-operative \\
\hline Patients & 7,701 & $4,017(52)$ & $3,684(48)$ \\
Women & $4,432(58)$ & $2,188(54)$ & $2,244(61)$ \\
Mean age (SD) & & & \\
$\quad$ All & $57(19)$ & 53 & 63 \\
$\quad$ Women & $63(18)$ & 58 & 69 \\
$\quad$ Men & $52(17)$ & 47 & 54 \\
TKR & & & \\
$\quad$ All & $340(4)$ & $202(5)$ & $138(4)$ \\
$\quad$ Women & $248(5)$ & $144(7)$ & $101(5)$ \\
$\quad$ Men & $95(3)$ & $58(3)$ & $37(3)$ \\
Years (SD) to TKRS & $2.1(2)$ & 2.2 & 2.0 \\
Mean age at TKRS (SD) & $65(12)$ & 62 & 69 \\
\hline
\end{tabular}

Table 2. Proportions of different follow-up events: patients requiring TKRS, patients without TKRS at follow-up end, and patients who died during follow-up. Values are count (\%)

\begin{tabular}{|c|c|c|c|c|c|c|}
\hline \multirow{2}{*}{$\begin{array}{l}\text { Follow-up } \\
\text { status }\end{array}$} & \multicolumn{6}{|c|}{ Years of follow-up } \\
\hline & 1 & 2 & 3 & 5 & 7 & 11 \\
\hline No TKRS & $7,199(94)$ & $6,857(89)$ & $6,594(86)$ & $6,242(81)$ & $6,057(79)$ & $5,907(77)$ \\
\hline TKRS & $120(2)$ & $220(3)$ & $263(3)$ & 307 (4) & $323(4)$ & $340(4)$ \\
\hline Dead & $382(5)$ & $624(8)$ & $844(11)$ & $1,152(15)$ & $1,321(17)$ & $1,454(19)$ \\
\hline
\end{tabular}

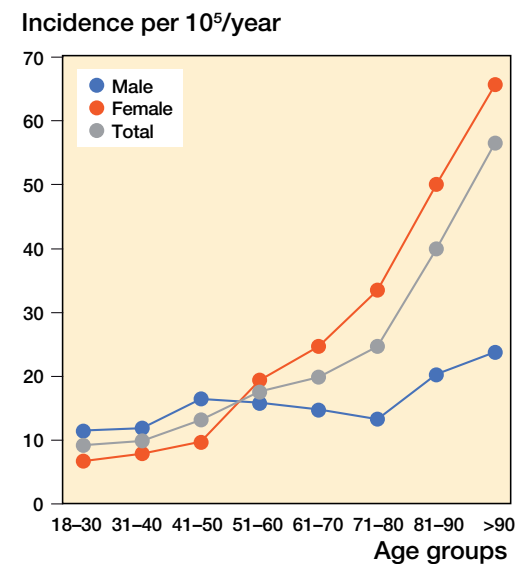

Figure 2. Annual incidence of proximal tibia fractures per 100,000/year.

analysis of risk for TKRS (Figure 1, Table 1). The annual incidence of TPF was $18 / 100,000$ (Figure 2). Mean follow-up time was 5.1 years (SD 3.1). Only $19(0.2 \%)$ patients had a bilateral fracture, but none of these patients needed TKRS. Of the laterality controls extracted from our hospital records, $0.6 \%$ of those with PTF underwent TKR on the contralateral side on average 6.6 years post-fracture.

During follow-up, TKRS was performed in

\section{Ethics, funding, and potential conflicts of interests}

Ethical approval was granted by National Institute for Health and Welfare (Dnro THL/1117/5.05.00/2019). The National Science Foundation funded the study. No conflicts of interests are declared by the authors.

\section{Results}

7,841 patients with 7,860 fractures were treated for PTF during the period 2009-2018. 7,701 of those were included in the
$340(4.4 \%)$ patients with prior PTF: 138 (3.7\%) in the nonoperatively treated group and $202(5.0 \%)$ in the operatively treated group (Table 1). Patients with prior TPF had higher risk of TKRS than the controls. The incidence of TKRS was highest during the first 2 years after fracture. Thereafter, it slowly declined while remaining elevated throughout the remainder of the follow-up (Table 2, Figure 3).

Mean age in the control group of 298,397 patients with unspecified abdominal pain was 50 years, and 186,541 (63\%) were women. TKR was performed in 4,238 (1.4\%) controls, of whom 2,927 (1.0\%) were women and 1,311 (0.4\%) men.
Cumulative risk of TKRS (\%)

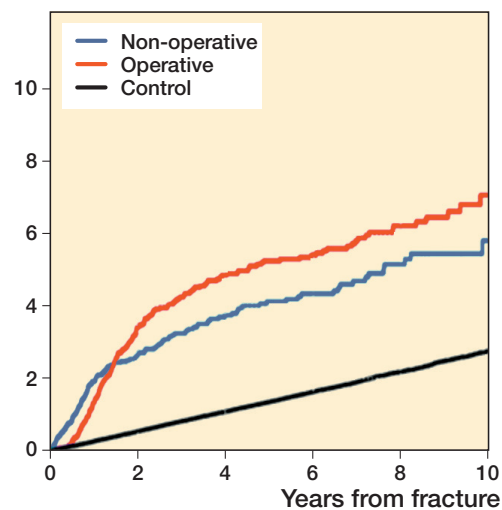

Cumulative risk of TKRS (\%)

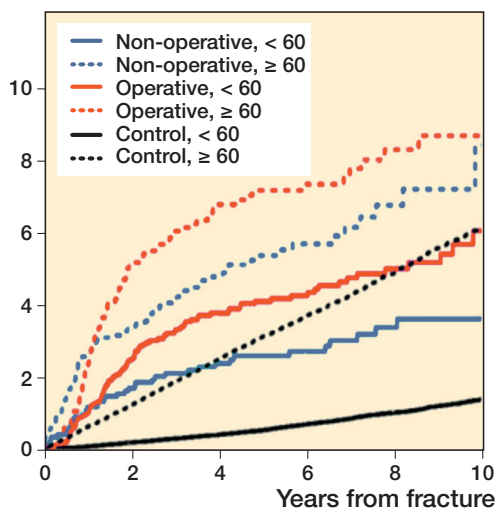

Cumulative risk of TKRS (\%)

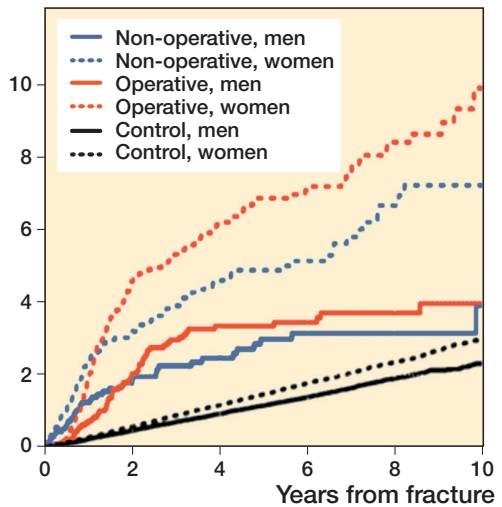

Figure 3. Kaplan-Meier curves showing TKRS risk: (left) after PTF, (middle) for patients under and over 60 years, and (right) for men and women, stratified by operative treatment, nonoperative treatment, and control group 

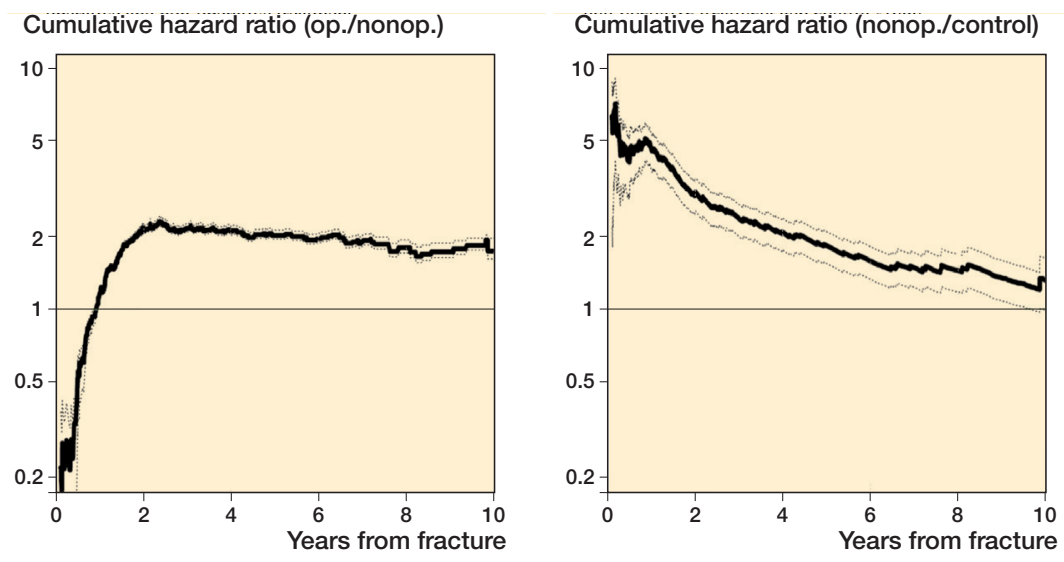

Figure 4. Age- and sex-adjusted cumulative treatment effect for TKRS between operative and nonoperative treatment and between nonoperative treatment and control group (comprising stomach pain patients without prior PTF) by time.

By the Cox modelling the risk of TKRS was 3.2-fold (HR 3.2 CI 2.8-3.7) in the operatively treated patients, and 1.8 -fold (HR 1.8, CI 1.6-2.2) in the nonoperatively treated patients compared with the reference group without PTF. Age (HR 1.05, CI 1.05-1.05) and female sex (HR 1.4, CI 1.3-1.4) were also risk factors for TKRS compared with the reference group.

However, the Kaplan-Meier curves (Figure 3) and the analysis of Schoenfeld residuals both indicated non-proportionality between the treatment groups. Hence a Cox model stratified by treatment group was fitted. The risk of TKRS after operative compared with nonoperative treatment increased about 2-fold during the first 2 years after surgery and remained at this elevated level throughout the follow-up. The risk of TKRS after nonoperative treatment in the PTF group compared with controls without PTF was highest during the first year after PTF. Thereafter it slowly decreased (Figure 4). The model concordance was 0.77 , and the global p-value of the Schoenfeld residuals was 0.55 , indicating proportional hazards of age and sex.

Mean time to TKRS was 2.2 (SD 2.0) years in the operatively treated group and 2.0 (SD 2.1) years in the nonoperatively treated group. Mean patient age at the time of TKRS was 65 (SD 12) years, whereas mean age at the time of TKR in the control group was 68 (SD 9.9) years.

\section{Discussion}

This register-based cohort study showed that the risk of TKRS was 3.2 times higher in patients with a prior operatively treated PTF and 1.8 times higher in patients with a nonoperatively treated PTF than in the population without prior PTF. The risk factors of TKRS were an operatively treated PTF, female sex, and high age.

The incidence of TPF in our data is between the levels reported in previous studies. Among the younger patients, the incidence was somewhat higher in men while among the older patients the incidence was significantly higher in women, as also previously reported (2-4).

While many studies have reported on the risk of TKRS after a tibial plateau or PTF, most are limited by the small size of the study population $(\mathbf{8}-11,14,15)$. Elsoe et al. (3) reported a 3.5-fold risk of TKRS after PTF in a sample where $5.7 \%$ of patients subsequently underwent TKRS. Wasserstein et al. (8) reported a higher, 5.3-fold risk in a sample where $7.3 \%$ of patients underwent TKRS during a 10-year follow-up. However, they studied only patients with tibial plateau fractures treated with ORIF, which explains the difference in rates between their study and ours. On the other hand, lower rates $(2.1 \%)$ of TKRS after ORIF treatment have also been reported (11). In our study, the risk of TKRS is in line with that reported by Elsoe et al., who also included patients with nonoperative treatment. The lower proportion $(4.3 \%)$ of patients who needed TKRS in our study may also be explained by the follow-up time, which was shorter than in the studies by Wasserstein et al. or Elsoe et al. $(\mathbf{8 , 9})$.

The major risk factors for TKRS were operative treatment, female sex, and high age. Comparison of operative with nonoperative treatment showed that the risk of TKRS increased in the operative group during the first 2 years and thereafter remained about double. Multi-fragmentary fractures have previously been reported as risk factors for reoperations after TPF (16). In our study, it is likely that the patients in the operative group sustained more displaced and more multi-fragmentary fractures than the patients in the nonoperative treatment group due to high-energy trauma in the younger patients or poor (osteoporotic) bone quality in the older patients. Hence, in cases with a more damaged articular surface, there is a greater likelihood of developing osteoarthritis and a higher risk of TKRS.

The association between TKRS and female sex may be explained by more osteoporotic bone quality leading to more damaged articular surface, which is usually difficult to reconstruct. An elevated risk of TKRS among women has also been reported previously $(\mathbf{8 , 9})$.

Higher age has also been reported to predict TKRS $(\mathbf{8 , 9})$, although conflicting findings exist (10). The higher risk of TKRS in older patients may also be explained by poor osteoporotic bone quality and a more damaged articular surface. In addition, in the case of painful knee, elderly patients are more likely to receive TKRS than younger patients. In our study, age increased the risk of TKRS.

\section{Advantages and limitations of the study}

Our study included the whole population of the country and compared one of the largest cohorts of patients with PTF and possible TKRS with a reference group 38 times larger. Moreover, the follow-up time was relatively long. This is the first 
large-volume study where patient age and sex and the method of treating PTF have been considered separately. Nevertheless, the study has some important limitations. Clinical information, fracture classification, intra- or extra-articular fractures, and patients' comorbidities and activities of daily living were not recorded in the registers, all factors which may have more effect on the need for TKRS than patient sex or age. Furthermore, the FHDR did not include information on the laterality of PTF or TKR; however, judging from the control group from our hospital database, the risk of observations of other sides without prior fracture was minimal. In any case, the true rate of TKRS on the same side as the PTF would be somewhat lower. The death of 1,454 (19\%) patients without need for TKRS during the follow-up must also be mentioned as a competing risk that may cause overestimation of the risk of TKRS. On the other hand, bias may have caused underestimation of the TKRS conversion rate. First, our study included patients treated for PTF as recently as December 2018, and hence the true rate of conversion to TKRS is likely to continue to increase with time in these recently operated patients. Moreover, the coverage of FAR was $94 \%$. A control group formed from hospital patients may differ from a control group selected from the general population and thus presents a potential risk of bias when comparing the risk ratios of target groups with those of a normal population. Last, it may be necessary to address possible erroneous data related to the health registers used.

\section{Conclusion}

During a mean follow-up of 5.1 years after PTF, 2.5-6.5\% of patients were treated with TKRS. These patients had a 1.8-3.2fold higher risk of TKRS than patients without PTF. Although operatively treated patients, women, and older patients were at higher risk of TKRS, other factors such as fracture classification, patients' comorbidities, and daily habits may have a greater effect on the need for TKRS. However, while the risk of later TKRS was low, it remained elevated throughout the follow-up, indicating that some patients develop long-lasting knee pain and decreased knee function after PTF.

\section{Supplementary data}

Appendix with ICD-10 codes is available as supplementary data.

Study design VT, KP, JP. Data collection VT, JH. Data analysis: VT, JH, KP. Manuscript preparation VT, KP, AT, JP.

Acta thanks Hans E Berg and Olof Wolf for help with peer review of this study.
1. Somersalo A, Paloneva J, Kautiainen H, Lönnroos E, Heinänen M, Kiviranta I. Increased mortality after lower extremity fractures in patients < 65 years of age. Acta Orthop 2016; 87: 622-5. doi: 10.1080/ 17453674.2016.1210533

2. Somersalo A, Paloneva J, Kautiainen H, Lönnroos E, Heinänen M, Kiviranta I. Incidence of fractures requiring inpatient care. Acta Orthop 2014; 85: 525-30. doi: 10.3109/17453674.2014.908340

3. Elsoe R, Larsen P, Nielsen N P H, Swenne J, Rasmussen S, Ostgaard S E. Population-based epidemiology of tibial plateau fractures. Orthopedics 2015; 38: 780-6. doi: 10.3928/01477447-20150902-55

4. Wennergren D, Bergdahl C, Ekelund J, Juto H, Sundfeldt M, Möller M. Epidemiology and incidence of tibia fractures in the Swedish Fracture Register. Injury 2018; 49: 2068-74. doi: 10.1016/j.injury.2018.09.008

5. Rademakers M V, Kerkhoffs G M M J, Sierevelt I N, Raaymakers E L F B, Marti R K. Operative treatment of 109 tibial plateau fractures: five- to 27-year follow-up results. J Orthop Trauma 2007; 21: 5-10. doi: 10.1097/BOT.0b013e31802c5b51

6. Schenker M L, Mauck R L, Ahn J, Mehta S. Pathogenesis and prevention of posttraumatic osteoarthritis after intra-articular fracture. J Am Acad Orthop Surg 2014; 22: 20-8. doi: 10.5435/JAAOS-22-01-20

7. Parkkinen M, Madanat R, Mustonen A, Koskinen S K, Paavola M, Lindahl J. Factors predicting the development of early osteoarthritis following lateral tibial plateau fractures: mid-term clinical and radiographic outcomes of 73 operatively treated patients. Scand J Surg 2014; 103: 25662. doi: $10.1177 / 1457496914520854$

8. Wasserstein D, Henry P, Paterson J M, Kreder H J, Jenkinson R. Risk of total knee arthroplasty after operatively treated tibial plateau fracture: a matched-population-based cohort study. J Bone Jt Surg 2014; 96 : 144-50. doi: 10.2106/JBJS.L.01691

9. Elsoe R, Johansen M B, Larsen P. Tibial plateau fractures are associated with a long-lasting increased risk of total knee arthroplasty: a matched cohort study of 7,950 tibial plateau fractures. Osteoarthritis Cartilage 2019; 27: 805-9. doi: 10.1016/j.joca.2018.12.020

10. Oladeji L O, Dreger T K, Pratte E L, Baumann C A, Stannard J P, Volgas D A, et al. Total knee arthroplasty versus osteochondral allograft: prevalence and risk factors following tibial plateau fractures. J Knee Surg 2019; 32: 380-6. doi: 10.1055/s-0038-1641593

11. Pinter Z, Jha A J, McGee A, Kyle P, Lee S, Dombrowsky A, et al. Outcomes of knee replacement in patients with posttraumatic arthritis due to previous tibial plateau fracture. Eur J Orthop Surg Traumatol 2020; 30: 323-8. doi: 10.1007/s00590-019-02575-4

12. Sund R. Quality of the Finnish Hospital Discharge Register: a systematic review. Scand J Public Health 2012; 40: 505-15. doi: 10.1177/ 1403494812456637

13. Wei G, Schaubel D E. Estimating cumulative treatment effects in the presence of nonproportional hazards. Biometrics 2008; 64: 724-32. doi: 10.1111/j.1541-0420.2007.00947

14. Mehin R, O'Brien P, Broekhuyse H, Blachut P, Guy P. Endstage arthritis following tibia plateau fractures: average 10-year follow-up. Can J Surg 2012; 55: 7-94. doi: 10.1503/cjs.003111

15. Scott C E, Davidson E, Macdonald D J, White T O, Keating J F. Total knee arthroplasty following tibial plateau fracture: a matched cohort study. Bone Jt J 2015; 97: 532-8. doi: 10.1302/0301-620X.97B4.34789

16. Wennergren D, Bergdahl C, Selse A, Ekelund J, Sundfeldt M, Möller M. Treatment and re-operation rates in one thousand and three hundred tibial fractures from the Swedish Fracture Register. Eur J Orthop Surg Traumatol 2021; 31: 143-54. doi: 10.1007/s00590-020-02751 


\section{Supplementary data}

APPENDIX

\begin{tabular}{ll}
\hline ICD-10 & Diagnosis/ Intervention \\
\hline Diagnosis code & \\
S82.1 & Proximal tibia fracture \\
NOMESCO code, Finnish version \\
NGJ62 & Internal fixation of fracture of knee or lower leg using plate and screws \\
NGJ64 & Internal fixation of fracture of lower leg using screws \\
NGJ70 & External fixation of fracture of knee or lower leg \\
NGJ99 & Other fracture surgery of knee or lower leg \\
NGB20 & Primary total prosthetic replacement of knee joint without patellar part \\
NGB30 & Primary total prosthetic replacement of knee joint without patellar part-connected prosthesis \\
NGB40 & Primary total prosthetic replacement of knee joint with patellar part-sliding prosthesis \\
NGB50 & Primary total prosthetic replacement of knee joint with patellar part-connected prosthesis \\
NGB99 & Other primary prosthetic replacement of knee joint, other or unspecified \\
\hline
\end{tabular}

